\title{
Immobilization of Gold Nanoparticles on Silanized Substrate for Sensors Based on Localized Surface Plasmon Resonance
}

\author{
Shuji Taue* \\ VBL, Collaborative Research Center, Hiroshima University, \\ 2-313 Kagamiyama, Higashi-Hiroshima 739-8527, Japan
}

\author{
Koji Nishida, Hiroyuki Sakaue, and Takayuki Takahagi \\ Graduate School of Advanced Sciences of Matter, Hiroshima University, \\ 1-3-1 Kagamiyama, Higashi-Hiroshima 739-8530, Japan
}

(Received 18 December 2006; Accepted 24 February 2007; Published 1 March 2007)

\begin{abstract}
We propose a detailed method of preparing immobilized gold nanoparticles on a substrate for application to dielectric sensors. We show the relationship between the amount of aminofunctional silane on the substrate and the absorbance caused by localized surface plasmon resonance of the gold nanoparticles. The amount of silane was changed by controlling rinsing time with distilled water. In the rinsing process, two features of dispersion for isolated nanoparticles on the substrate were observed. One was a high density of nanoparticles, but with some of them incompletely isolated; the other was a low density of nanoparticles, but all completely isolated. To confirm the suitability for sensor applications, we measured the absorption spectra of the substrates surrounding media of various dielectric constants. [DOI: 10.1380/ejssnt.2007.74]
\end{abstract}

Keywords: Scanning electron microscopy (SEM); X-ray photoelectron spectroscopy; Visible/ultraviolet absorption spectroscopy; Adhesion; Nanoparticles

\section{INTRODUCTION}

Nanoparticles of noble metals, like gold or silver, produce a strong electric field enhancement in visible light. This phenomenon is due to the localized surface plasmon resonance (LSPR) of free electrons in the metal. Since the resonance condition depends on the dielectric constant of the surrounding medium, there has been much research investigating the application of this phenomenon to various sensors. These sensors are typically constructed of gold nanoparticles immobilized on the substrate, together with an optical setup for spectrophotometry. For example, this kind of system can measure the thickness of dielectric films [1] and can detect the biomolecule streptavidin [2]. Furthermore, it has been reported that these sensor systems can probe the affinity between biological molecules using an optical fiber [3].

In these sensors, aminofunctinal silane is often used as a coupling agent between the gold nanoparticles and the substrate. To deposit a sufficient amount of silane homogeneously on the substrate, the cleaned substrate is immersed in $10 \%(\mathrm{v} / \mathrm{v})[1,2]$ silane solution for 10 or 15 minutes $[1-3]$. The silane forms hydrogen bonds with hydroxyl groups on the substrate. After the surplus silane on the surface is rinsed off, the substrate is dried and baked at $120^{\circ} \mathrm{C}[2,3]$ to form siloxane bonds between the silane and the surface. A monolayer of silane remains adhered to the substrate. Finally, the substrate is immersed in an aqueous solution of gold nanoparticles. The nanoparticles in the solution are negatively charged by modification of carboxyl groups, and the amino groups of the aminofunctional silanes adhered on the substrate are positively charged. The electrostatic attraction thus draws

*Corresponding author: taue@ieee.org the nanoparticles to the substrate, and the amino groups and the carboxyl groups bond to immobilize the nanoparticles on the substrate. The charge of the nanoparticles results in a repulsive force between the nanoparticles in the solution and those on the substrate. As a result, the gold nanoparticles are immobilized on the substrate in isolation, and the optical properties of these isolated nanoparticles are similar to those of colloidal solution. Therefore, the dielectric constant or refractive index of a medium surrounding the substrate can easily be measured by using LSPR of the gold nanoparticles immobilized on the substrate.

It is important to immobilize many nanoparticles in isolation, because the greater the number of nanoparticles the better the signal-to-noise ratio of the sensor. It is thought that a greater amount of silane could immobilize many nanoparticles on the substrate. However, the silane causes aggregation of the gold nanoparticles if it elutes from the substrate to the solution of gold nanoparticles. Even if it does not elute, there is a possibility that surplus silane on the substrate immobilizes the nanoparticles and causes them to stick together. This aggregation of the nanoparticles on the substrate causes a change in the absorbance spectrum [4]. The peak wavelength of LSPR for nanoparticles immobilized in isolation is approximately $520 \mathrm{~nm}$, but that for aggregated nanoparticles is nearly $650 \mathrm{~nm}$. This phenomenon causes a corresponding reduction in resolution of sensors that use the shift of LSPR wavelength as a signal. Hence, it is important not to aggregate the nanoparticles, but to immobilize them in isolation as much as possible during the substrate preparation process.

The aim of this work is to immobilize gold nanoparticles on a substrate in isolation. To immobilize many nanoparticles, we used a $10 \%(\mathrm{v} / \mathrm{v})$ solution of aminofunctionl silane and immersed the substrate in the colloidal solution for a sufficient length of time. We controlled the rinsing 


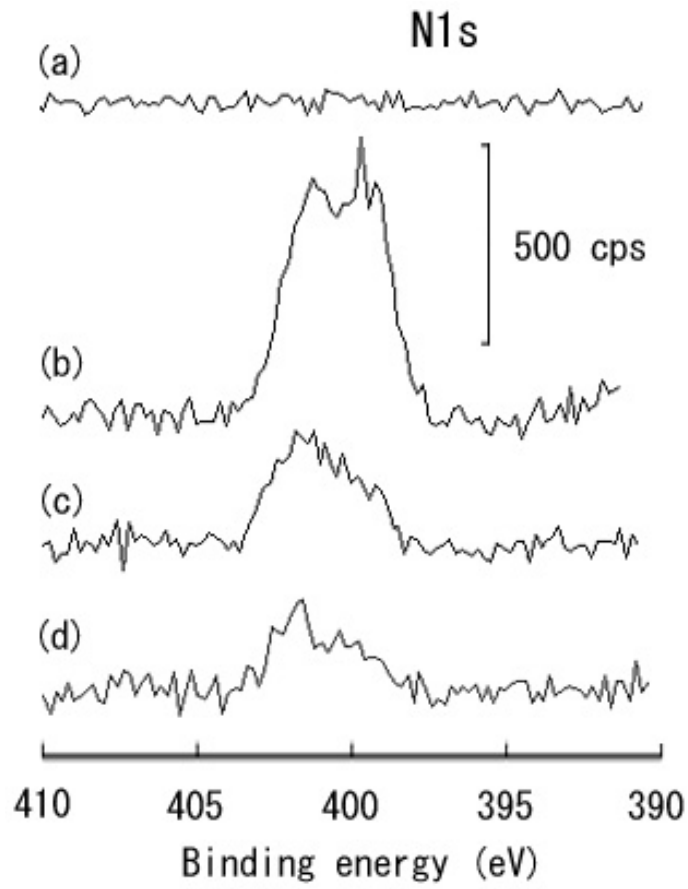

FIG. 1: XPS narrow spectra of the nitrogen signal of the substrate (a) before immersion in APTES solution, and after immesion and rinsing for (b) 15 minutes, (c) 30 minutes, and (d) 60 minutes.

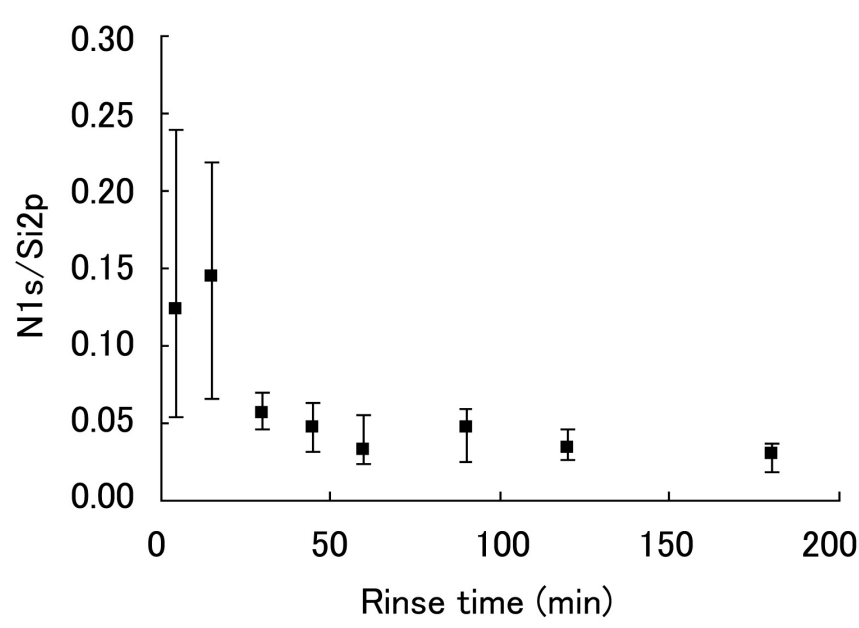

FIG. 2: Relationship between the rinse time of 5, 15, 30, 45, $60,90,120$, and 180 minutes and $\mathrm{N} 1 s / \mathrm{Si} 2 p$ ratio.

time to remove surplus silane in order not to cause the aggregation of the nanoparticles. By evaluating the absorbance at the resonance wavelength, we studied the relationship between the amount of silane and the quantity of nanoparticles immobilized on the substrate. To confirm the suitability for sensor applications, we measured the absorption spectra of gold nanoparticles immobilized on a substrate immersed in solvents of various dielectric conditions.

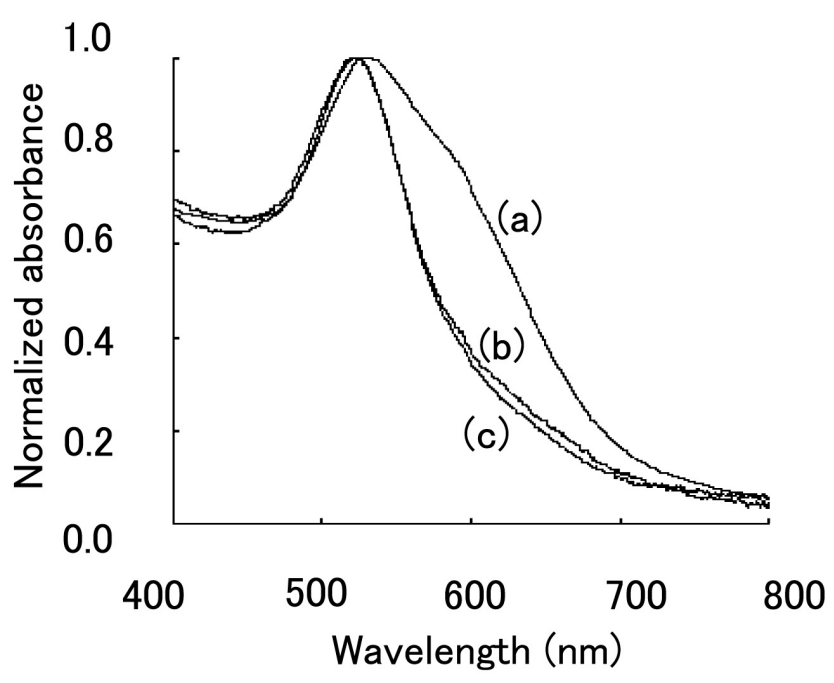

FIG. 3: UV-visible absorption spectra of the substrates rinsed for (a) 5 minutes, (b) 15 minutes, and (c) 60 minutes.

\section{EXPERIMENTAL}

All glassware used in these experiments was cleaned by immersing in $0.5 \%(\mathrm{v} / \mathrm{v})$ aqueous solution of ammonium peroxodisulfate and boiling for 20 minutes. It is important to use clean equipment to prevent aggregation of the nanoparticles. Gold nanoparticles were synthesized by a reduction of hydrogen tetrachloroaurate (III) tetrahydrate $\left(\mathrm{HAuCl}_{4} \cdot 4 \mathrm{H}_{2} \mathrm{O}\right)$ by trisodium citrate dihydrate $\left(\mathrm{C}_{6} \mathrm{H}_{5} \mathrm{O}_{7} \mathrm{Na}_{3} \cdot 2 \mathrm{H}_{2} \mathrm{O}\right)$ and tannic acid $\left(\mathrm{C}_{14} \mathrm{H}_{10} \mathrm{O}_{9}\right)$ [5]. Trisodium citrate dihydrate in a concentration of $2.8 \times 10^{-4} \mathrm{~mol} / \mathrm{l}$ and tannic acid in a concentration of $1.6 \times 10^{-5} \mathrm{~mol} / \mathrm{l}$ were mixed and heated to $60^{\circ} \mathrm{C}$ on a hot plate. This reducing mixture was rapidly added to $2.4 \times 10^{-4} \mathrm{~mol} / \mathrm{l}$ hydrogen tetrachloroaturate (III) tetrahydrate solution that was also heated to $60^{\circ} \mathrm{C}$, and stirred to the end of the procedure. After the color of the solution turned red, the solution was boiled for 15 minutes and then cooled to room temperature. The resulting gold nanoparticles were found to have an average diameter of $11 \mathrm{~nm}$, observed by a scanning electron microscope (SEM; Hitachi, S-5000).

Quartz substrates of $1 \mathrm{~mm}$ thickness were cut out to dimensions $9 \mathrm{~mm} \times 24 \mathrm{~mm}$. The substrates were cleaned using UV irradiation to oxidize them for 10 minutes and immersed in $\mathrm{H}_{2} \mathrm{O}_{2}$ mixed in a $1: 3$ ratio with $\mathrm{H}_{2} \mathrm{SO}_{4}$ for 10 minutes. The substrates were subsequently immersed in a $10 \%(\mathrm{v} / \mathrm{v})$ aqueous solution of 3-aminopropyltriethoxysilane (APTES) as an aminofunctional silane for 30 minutes. From our research, this concentration of APTES solution and immersion time was enough to deposit sufficient APTES on the substrate. There has been some research on immobilizing nanoparticles on substrates using a silane solution with a lower density without the rinsing process [6], but our research indicates that, at some areas of the substrate, the quantity of nanoparticles immobilized with this method is low and the distribution is inhomogeneous. It is thought that electrostatic attraction between amino groups of the silane and the surface only occurs if there is no physisorption of 
(a)

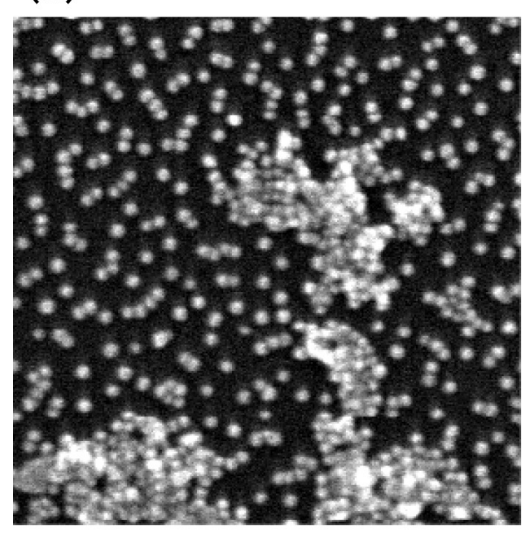

(b)

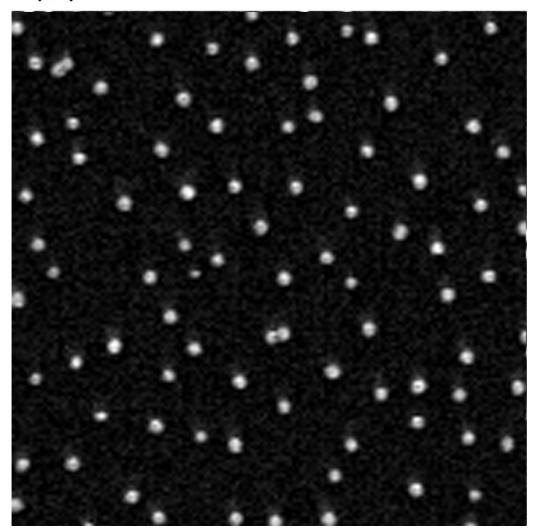

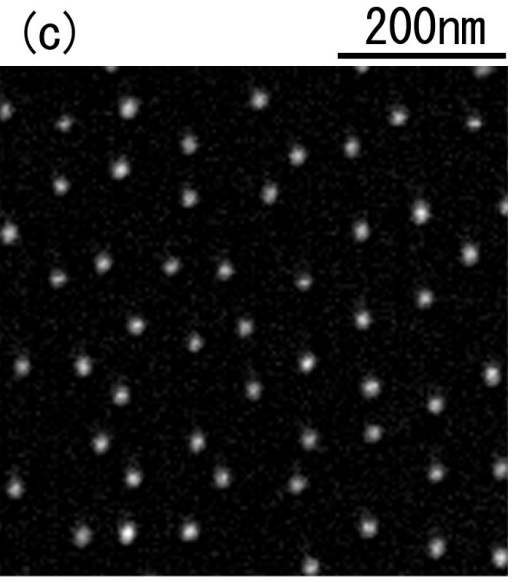

FIG. 4: SEM images of gold nanoparticles immobilized on silicon substrate rinsed for (a) 5 minutes, (b) 15 minutes, and (c) 60 minutes.

APTES on the substrate [7]. After immersion, the substrates were dipped in distilled water filled in a beaker to rinse off surplus APTES. The rinse time was varied from 5 to 180 minutes. Distilled water slowly and continuously added to the beaker and overflowed to prevent deposition of other substances present on the water surface when the substrate was removed from the water. The substrate was then dried in air for 10 minutes. After drying, the substrate was baked at $120^{\circ} \mathrm{C}$ for 10 minutes in a glass jar that was pre-heated on a hot plate, and its temperature was monitored using a thermometer. From our previous research, this baking time was enough to prevent elution of APTES from the sufficiently rinsed substrate. The amount of APTES on the substrate was obtained from the relative amounts of nitrogen and silicon measured with an X-ray photoelectron spectrometer (XPS, Perkin Elmer, Phi 1600) at a measuring area of $0.8 \mathrm{~mm} \times 2$ $\mathrm{mm}$ and an exit angle of $45^{\circ}$. Binding energies of all XPS spectra were calibrated using the $\mathrm{Si} 2 p$ signal at $103.5 \mathrm{eV}$.

The silanized substrate was then immersed in an aqueous solution of the gold nanoparticles for 24 hours. From our research, the quantity of nanoparticles immobilized on the substrate did not increase with an immersion time above 12 hours. After immersion, the substrates were dipped in distilled water for 1 minute to remove nanoparticles that were not immobilized.

To estimate the absorption spectrum due to LSPR of the gold nanoparticles immobilized on the substrate, we used a UV-visible spectrophotometer (Shimadzu, UV$2500 \mathrm{PC}$ ). In our measurements, after a sample cell was filled with a solvent, the substrate was immersed in it to be perpendicular to the optical axis, which was vertical. We used distilled water, ethanol, 1-hexanol, carbon tetrachloride, and toluene as the solvent. The absorbance of gold nanoparticles at all wavelengths was given by $\log _{10} I_{0} / I$, where $I_{0}$ is the transmitted light intensity through the solvent and the substrate without nanoparticles, and $I$ is the transmitted light intensity through the solvent and the substrate with nanoparticles. We also used SEM to observe the isolation feature of the gold nanoparticles immobilized on the substrate. For this observation, we had to use a silicon substrate as a substitute for a quartz one because conductivity was required for the
SEM observation. Silicon is conductive and has a similar surface composition to quartz. We conducted the same procedure as described above on the silicon substrates.

\section{RESULTS AND DISCUSSION}

N1s narrow spectra of nitrogen measured by XPS are shown in Fig. 1. Figure 1(a) shows the spectrum obtained from substrates before immersion in the APTES solution, and Figs. 1(b), (c), and (d) show the spectra obtained from the substrates after immersion in the APTES solution and rinsed for 15, 30, and 60 minutes, respectively. APTES has one atom of nitrogen in a molecule. The nitrogen signal did not exist on the substrate before immersion in APTES solution. After immersion, nitrogen signals were obtained at a binding energy of about $400 \mathrm{eV}$ and decreased gradually with the rinse time. The ratio of nitrogen and silicon (N1s/Si2p) was used to evaluate the relative amount of APTES on the substrate. APTES also has one atom of silicon in a molecule, but almost all of the silicon signals detected by XPS were from the substrate.

The relationship between rinse time and $\mathrm{N} 1 s / \mathrm{Si} 2 p$ ratio is shown in Fig. 2. The $\mathrm{N} 1 s / \mathrm{Si} 2 p$ ratios at 5 and 15 minutes of rinse time were about 0.14 and exhibited significant variation from preparation to preparation. At 30 minutes of rinse time, the ratio decreased considerably and settled. At over 60 minutes of rinse time, the ratio was about 0.04 . These results indicate that a significant amount of APTES deposited on the substrate eluted with rinsing for as long as 30 minutes. A certain amount of the APTES did not elute with rinsing. It is thought that eluted APTES was physisorbed to the substrate or to APTES on the substrate, and the remaining APTES was chemisorbed to the substrate.

To investigate the immobilization feature of the gold nanoparticles on the substrate, absorption spectra of the substrates rinsed for 5, 15, and 60 minutes and immersed in the solution of nanoparticles are shown in Figs. 3(a), (b), and (c), respectively. SEM images of the nanoparticles immobilized on the silicon substrates rinsed for 5, 15, and 60 minutes and immersed in the solution of nanoparticles are shown in Figs. 4(a), (b), and (c), re- 


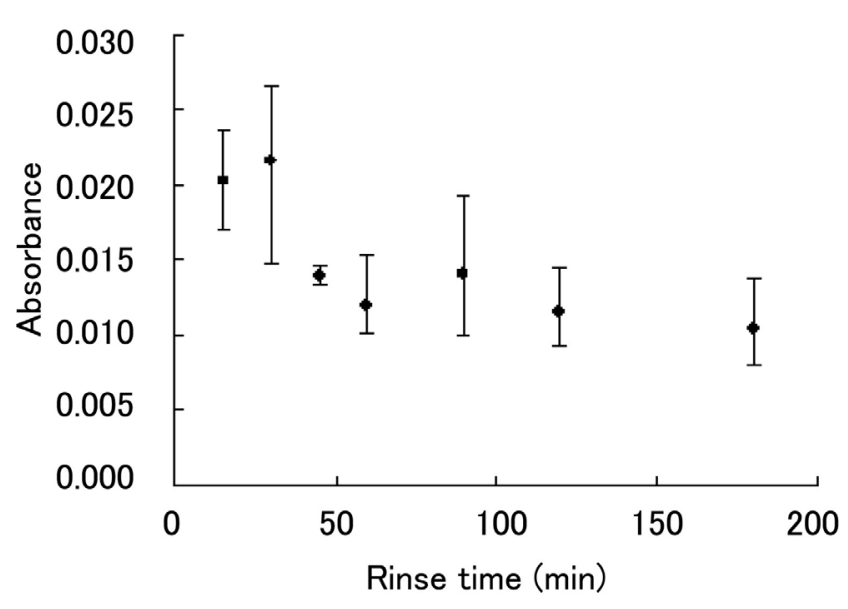

FIG. 5: Relationship between the rinse time of 15, 30, 45, 60, 90, 120, and 180 minutes and the absorbance intensity.

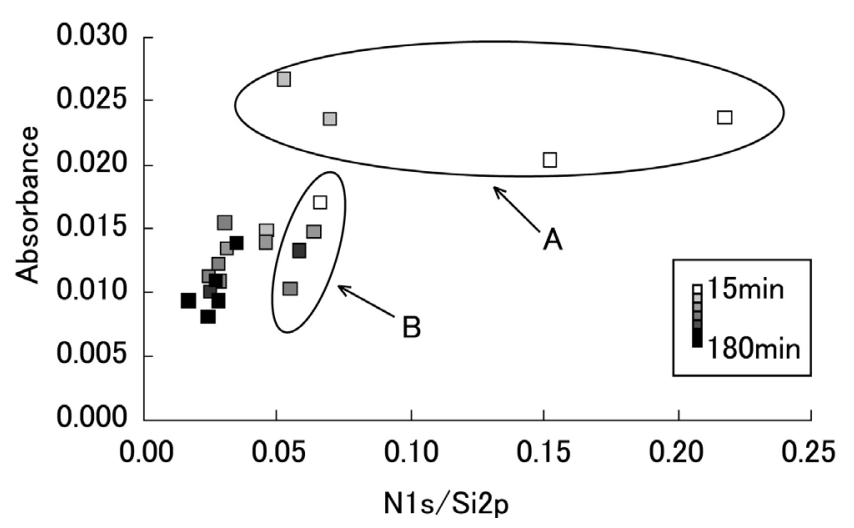

FIG. 6: Relationship between N1s/Si2p and the absorbance intensity.

spectively. Some of the substrates rinsed for 5 minutes showed a double peak spectrum at about $530 \mathrm{~nm}$ and 600 $\mathrm{nm}$, as shown in Fig. 3(a), and immobilized nanoparticles were aggregated on the substrate, as shown in Fig. 4(a). The solution of the nanoparticles also changed color from red to bluish purple. Only the substrate rinsed for 5 minutes showed the nanoparticle aggregation and double-peak spectrum. Furthermore, the intensity of the additional peak at about $600 \mathrm{~nm}$ was different in every preparation. Aggregation of the nanoparticles could be prevented by rinsing the substrates for over 15 minutes. The substrates rinsed for 15 and 60 minutes showed little difference in the shape of their absorbance spectra, as shown in Figs. 3(b) and (c). Both peak wavelengths, due to LSPR, were $522 \mathrm{~nm}$. As shown in Figs. 4(b) and (c), most nanoparticles were immobilized in isolation on both substrates. The numbers of nanoparticles counted in a 1$\mu \mathrm{m}^{2}$ area in the SEM images were about 230 and 160 on the substrates rinsed for 15 and 60 minutes, respectively. Although the number of nanoparticles on the substrate rinsed for 15 minutes was higher, some nanoparticles were stuck to each other, as shown in Fig. 4(b).

The relationship between the rinse time and the absorbance of the spectrum is shown in Fig. 5. The absorbance intensities at 15 and 30 minutes of rinse time<smiles>CO[Si](CCCN)(OC)OC</smiles>

(a)<smiles>CO[Si]1(OC)CCC[NH2+]O1</smiles>

(b)
FIG. 7: Schematic representations of the structure of (a) normal and (b) cyclic APTES.

(a)
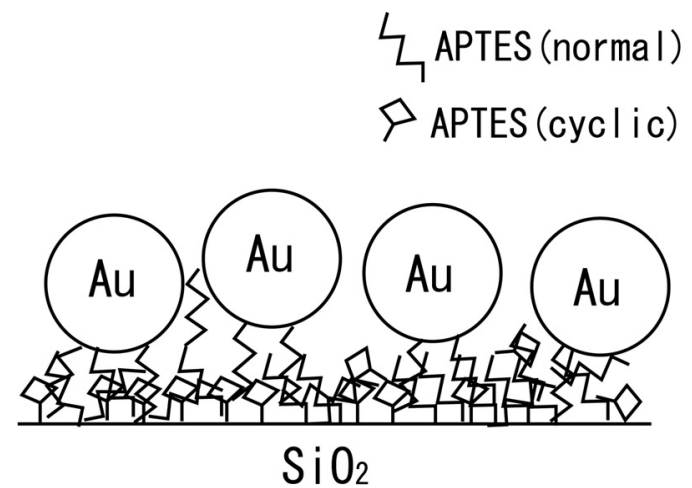

(b)

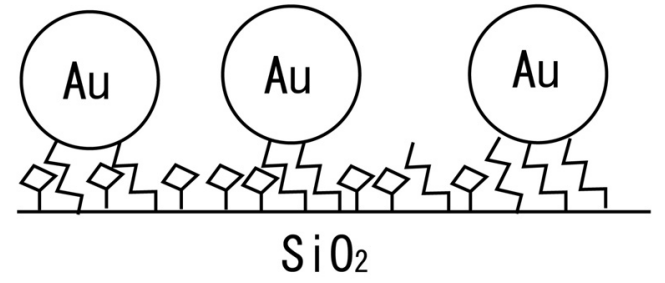

FIG. 8: Schematic representations of immobilization of gold nanoparticles on the substrate (a) with the surplus APTES, (b) without surplus APTES.

were about 0.02 . The $\mathrm{N} 1 s / \mathrm{Si} 2 p$ ratios at 15 and $30 \mathrm{~min}-$ utes of rinse time were quite different, but there was little difference between the absorbance intensities. At over 60 minutes of rinse time, the absorbance intensities reduced to about 0.013 .

The relationship between the $\mathrm{N} 1 s / \mathrm{Si} 2 p$ ratios and the absorbance intensities are plotted in Fig. 6. The gray scale in each plot indicates the rinse time. Almost all of the plots indicate that the absorbance intensity increased according to the $\mathrm{N} 1 s / \mathrm{Si} 2 p$ ratio, but the absorbance intensities stopped increasing at about 0.025 , as shown in the region A of Fig. 6. This region suggests that the nanoparticles immobilized by APTES do not exceed a certain quantity without aggregation. It is thought that the 

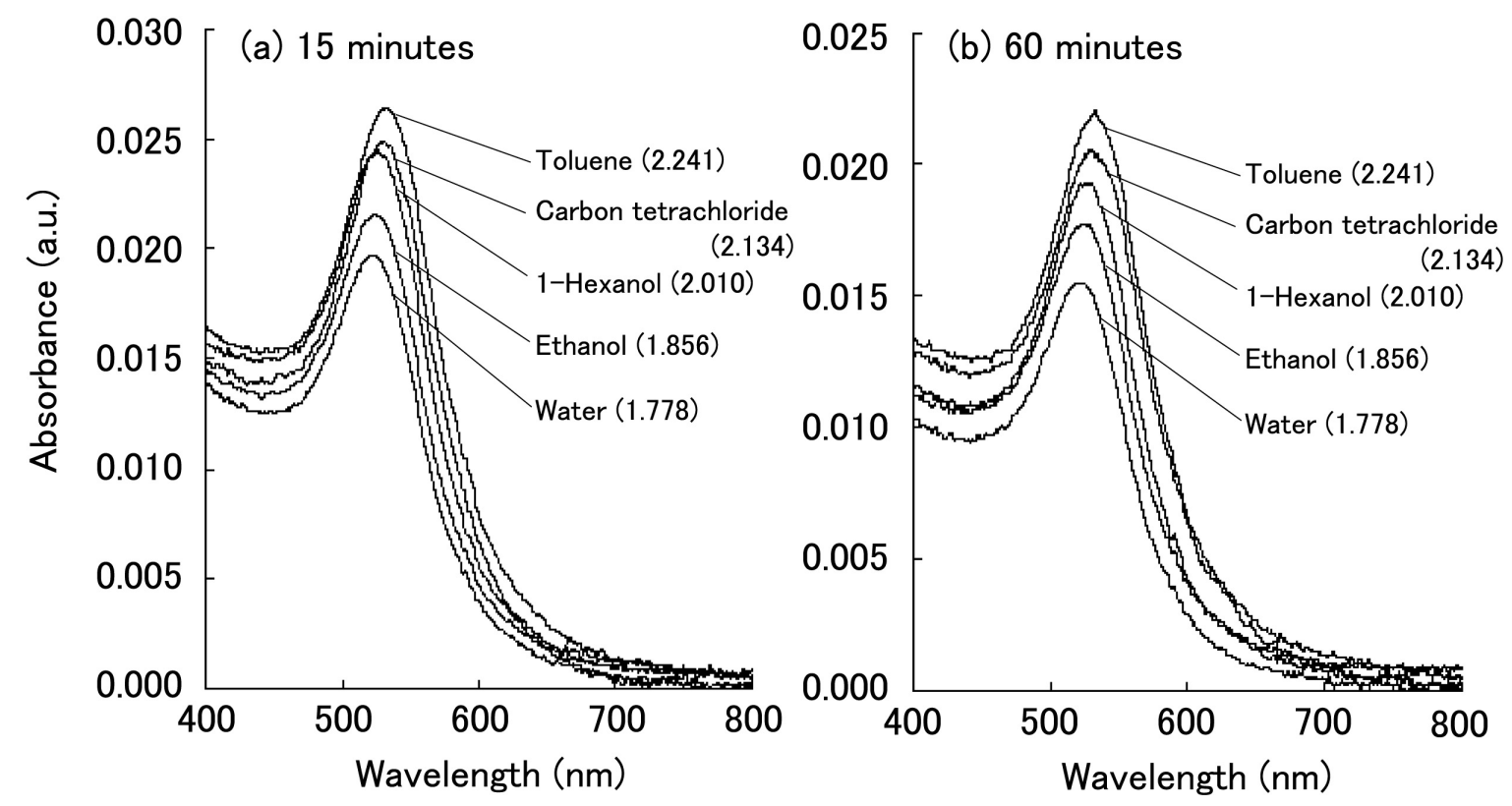

FIG. 9: Absorption spectra of gold nanoparticles immobilized on the substrate rinsed for (a) 15 minutes and (b) 60 minutes after immersion in various solvents.

quantity is determined by the electrostatic repulsion of each nanoparticle in colloidal solution, even if a greater amount of APTES attracts more nanoparticles. This repulsion force prevents immobilizing nanoparticles much closer together. This phenomenon may therefore be useful in improving the fabrication reproducibility of sensor probes. However, there was a reduction in the absorbance intensity versus $\mathrm{N} 1 s / \mathrm{Si} 2 p$ ratio, as shown in region $\mathrm{B}$ of Fig. 6. The reason of this reduction was elution of APTES in water droplets on the substrate during the drying process. For the surface measurement by XPS, we did not use artificial drying to prevent the deposition of other substances. Heterogeneous adhesion of APTES occurred on the substrate. Therefore, this reduction in absorbance intensity occurred at some substrates rinsed for below 60 minutes. As a result, this heterogeneity led to local differences of the quantity of nanoparticles immobilized on the substrate. In addition, there was a difference in the measurement areas used in the XPS and UV-visible spectrophotometer, which led to a reduction of the absorbance intensity.

The absorbance intensities at over 60 minutes of rinse time also settled to 0.013. The amount of APTES did not decrease below a certain amount for over 60 minutes of rinse, as shown above. The quantity of nanoparticles immobilized on the substrate was determined by the settled APTES amount. This behavior is also useful for improving fabrication reproducibility. However, the absorbance intensity was less than that at 15 or 30 minutes of rinse time. One possible reason is that the positive charge of the amino groups decreased as the amount of APTES on the substrate decreased, thus also reducing the force required to attract the nanoparticles to the substrate.

Not all of the APTES could immobilize the nanoparticles. From previous research, APTES forms normal and cyclic structures in aqueous solution, as shown in Figs. $7(\mathrm{a})$ and (b), respectively $[8,9]$. An amino group of the cyclic structure forms an internal hydrogen bond with hydrolyzed silanol. Two peaks at the binding energy of nitrogen are clearly shown in Fig. 1. The binding energies of the two peaks are about 399 and $401 \mathrm{eV}$. To assign both peaks, we measured correlation samples of $\mathrm{BuNH}_{2}$ and $\mathrm{H}_{3} \mathrm{~N}^{+} \mathrm{CHRCOO}^{-}$, and we assigned the peaks of 399 and $401 \mathrm{eV}$ to free amine $\left(\mathrm{NH}_{2}\right)$ and protonated amine $\left(\mathrm{NH}_{3}^{+}\right)$, respectively. In the XPS measurement, the free amine and the protonated amine correspond to the normal and cyclic structures of APTES, respectively. When APTES adhered to a substrate is immersed in a colloidal solution, the free amine also changes to protonated amine with the normal APTES structure. This amine can attract and immobilize nanoparticles. On the other hand, the protonated amine with the cyclic APTES structure cannot immobilize the nanoparticles because the structure does not change to the normal one even in the colloidal solution. From these results, we show the schematic representation of the immobilization of nanoparticles with and without surplus APTES in Figs. 8(a) and (b), respectively. As shown in Fig. 8(a), much surplus APTES existed on the substrates rinsed for 15 or 30 minutes. Many nanoparticles in the solution were attracted by the strong positive charge of the large amount of APTES on the substrate. Almost all of immobilized nanoparticles were isolated by the electrical repulsion, but the SEM images show that a few nanoparticles stuck to each other. On the other hand, as shown in Fig. 8(b), no surplus APTES adhered on the substrate rinsed for over 60 minutes. The attraction force was less due to the reduced amount of APTES. The nanoparticles were thus immobilized by normal APTES structure and were well isolated from each other.

Finally, we show the difference in LSPR properties due to the quantity of gold nanoparticles immobilized on the substrate. The substrates were prepared as described above and rinsed for 15 and 60 minutes. However, the drying process was changed to $\mathrm{N}_{2}$ flashing to prevent het- 


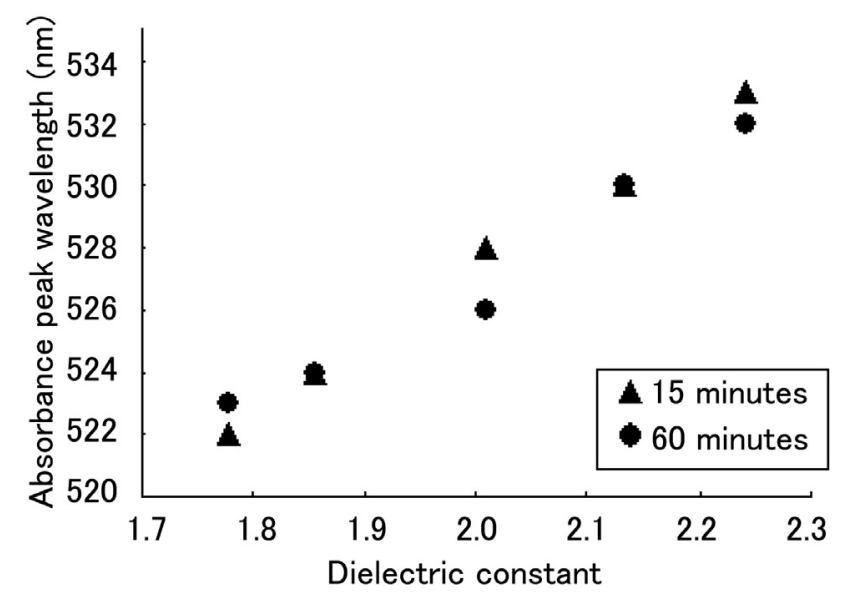

FIG. 10: Relationship between the wavelength at the resonance peak and the dielectric constant of the solvent.

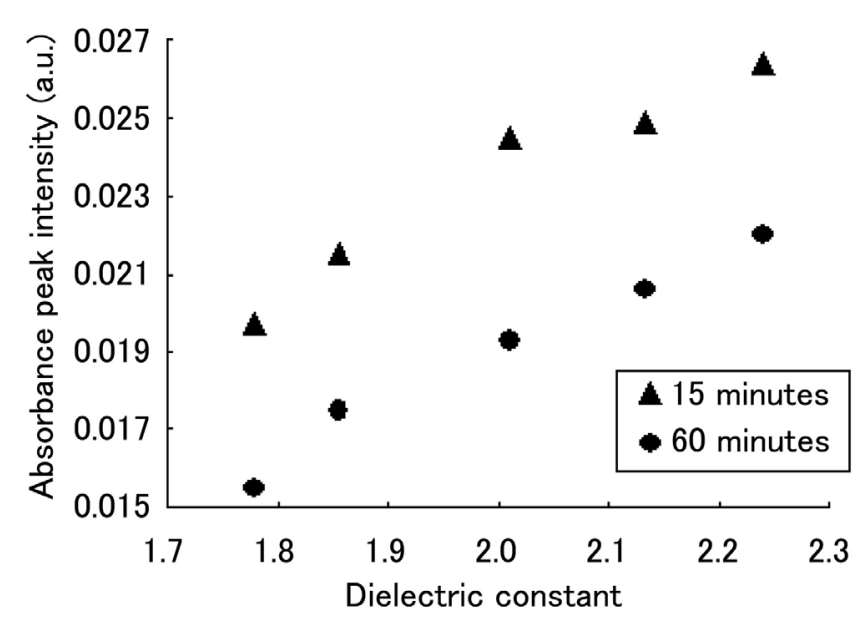

FIG. 11: Relationship between the absorbance intensity at the resonance peak and the dielectric constant of the solvent.

erogeneous immobilization of the nanoparticles.

Figures 9(a) and (b) show absorption spectra of the substrates rinsed for 15 and 60 minutes, respectively. The spectra were measured for various surrounding media. The dielectric constants of the surrounding media, measured at a wavelength of $589.3 \mathrm{~nm}$, are also shown. The absorbance spectra of each substrate show single peaks between 520 and $550 \mathrm{~nm}$ caused by LSPR. The wavelength and intensity of the absorbance peaks changed with the dielectric constant of the surrounding medium.

To examine the difference of the absorbance peaks between two substrates, the relationship between the peak wavelength due to LSPR and the dielectric constant of the surrounding medium is shown in Fig. 10. The resonance wavelength linearly increased as the dielectric constant increased, and there was little difference between the two substrates. These results agree with the extinction cross section of an isolated spherical gold nanoparticle in a uniform medium calculated by Mie's theory. It is thought that this behavior is well suited for sensors using disposable probes, because differences from lot to lot can be prevented.

The relationship between the absorbance intensity at the resonance wavelength and the dielectric constant is shown in Fig. 11. The absorbance intensity at the resonance wavelength also linearly increased as the dielectric constant increased. The variations in absorbance with respect to the dielectric constant were similar in the two substrates, but the absolute absorbance levels at all dielectric constants were different. The substrate rinsed for 15 minutes exhibited an absorbance intensity about 0.004 higher than that of the substrate rinsed for 60 minutes. This higher absorbance intensity will lead to higher signalto-noise ratio for sensors using absorbance intensity as a signal.

\section{CONCLUSIONS}

We focused attention on the rinsing process in existing fabrication method. To prevent aggregation of the nanoparticles, a rinse time of over 15 minutes was required. The substrates rinsed for 15 and 30 minutes exhibited high, nearly constant absorbance intensities at the resonance wavelength, although the amount of APTES adhered on the substrates varied greatly. The substrate rinsed for over 60 minutes exhibited a nearly constant but lower absorbance intensity because of the constant amount of APTES. Some nanoparticles immobilized on the substrate rinsed for 15 minutes were not isolated, but the optical properties, possibly used in a sensor, were the same as for isolated immobilization. Our proposed method indicates the possibility of a reproducible process to immobilize many isolated nanoparticles on a substrate. It is thought that the increased absorbance intensity will improve the resolution of sensor devices based on this technology.
[1] T. Okamoto, I. Yamaguchi, and T. Kobayashi, Opt. Lett. 25, 372 (2000).

[2] N. Nath and A. Chilkoti, Anal. Chem. 74, 504 (2002).

[3] K. Mitsui, Y. Handa, and K. Kajikawa, Appl. Phys. Lett. 85, 4231 (2004).

[4] S. Huang, K. Minami, H. Sakaue, S. Shingubara, and T. Takahagi, J. Appl. Phys. 92, 7486 (2002).

[5] G. Tsutsui, S. Huang, H. Sakaue, S. Shingubara, and T. Takahagi, Jpn. J. Appl. Phys. 40, 346 (2001).

[6] T. Sato, D. G. Hasko, and H. Ahmed, J. Vac. Sci. Technol.
B 15, 45 (1997).

[7] E. T. Vandenberg, L. Bertilsson, B. Liedberg, K. Uvdal, R. Erlandsson, H. Elwing, and I. Lundström, J. Colloid Interface Sci. 147, 103 (1991).

[8] C. Chiang, H. Ishida, and J. L. Koenig, J. Coll. Int. Sci. 74, 396 (1980).

[9] D. Suryanarayana and K. L. Mittal, J. Appl. Poly. Sci. 29, 2039 (1984). 